\title{
LiNEAR DifFERENTIAL AlgEBRAIC EQUATIONS WITH CONSTANT COEFFICIENTS
}

\author{
${ }^{1} \mathrm{M}$. Sahadet Hossain and ${ }^{2} \mathrm{M}$. Mostafizur Rahman \\ ${ }^{1}$ Deparment of Mathematics, Chemnitz University of Technology, Germany \\ ${ }^{2}$ Department of Information Engineering and Science, University of Trento, Trento, Italy \\ E-mail:mohh@hrz.tu-chemnitz.de and mohammed.rahman@studenti.unitn.it.
}

\begin{abstract}
Differential-algebraic equations (DAEs) arise in a variety of applications. Their analysis and numerical treatment, therefore, plays an important role in modern mathematics. The paper gives an introduction to the topics of DAEs. Examples of DAEs are considered showing their importance for practical problems. Some essential concepts that are really essential for understanding the DAE systems are introduced. The canonical forms of DAEs are discussed widely to make them more efficient and easy for practical use. Also some numerical examples are discussed to clarify the existence and uniqueness of the system's solutions.
\end{abstract}

Keywords: differential-algebraic equations, index concept, canonical forms.

\section{Introduction}

Differential-algebraic equations (DAEs) have wide applications in the modeling and simulation of constrained dynamical systems in numerous applications, such as mechanical multibody systems[1][2], electrical circuit simulation[2][3], chemical engineering, control theory[1][3][4], fluid dynamics, and many other areas. The dynamical behavior of physical processes is usually modeled via differential equations. But if the states of the physical system are in some ways constrained, like for example by conservation laws such as Kirchhoff's laws in the electrical networks[3], or by position constraints such as the movement of mass points on a surface[5], then the mathematical model also contains algebraic equations to describe these constraints. Such systems, consisting of both differential and algebraic equations are called differential-algebraic systems, algebrodifferential systems, or implicit differential equations.

A Differential-Algebraic Equation (DAE) is, essentially, an Ordinary Differential Equation (ODE) of the type
$F(t, x, \dot{x})=0$

with $F: I \times X^{n} \times X^{n}=X^{m}, \quad$ where $I \subseteq P$ and $m, n \in N$ that cannot be solved explicitly for the derivative $\dot{x}$. The name comes from the fact that in some cases they can be reduced to two-part system: A usual differential system plus an algebraic part, that

is $\left\{\begin{array}{l}\dot{x}=f(t, x, z) ; \\ 0=g(t, x, z) .\end{array}\right.$

Here the ODE (2) for $x(t)$ depends on additional algebraic variable $z(t)$, and the solution is forced in addition to satisfy the algebraic constraints given with it. The system (2) is called a semi explicit system of differential-algebraic equations (DAEs).

In comparison with ODEs, these equations present at least two major difficulties: the first lies in the fact that it is not possible to establish general existence and uniqueness results, due to their more complicate structure; the second one is that DAEs do not regularize the input, since solving them typically involves differentiation in place of integration.[1]-[6].

\section{Examples of Differential-Algebraic Equations}

Modeling with DAEs plays a vital role, among others, for constrained mechanical systems, electrical circuits and chemical reaction kinetics. We will discuss here one important application of DAEs in constrained mechanical systems.[1]

\subsection{Constrained mechanical systems}

Consider the mathematical pendulum in figure 1. Let $\mathrm{m}$ be the pendulum's mass which is attached to a rod of length $l$. In order to describe the pendulum in Cartesian coordinates we write down the potential energy [1][7] 


$$
U(x, y)=m g h=m g l-m g y
$$

where $(x(t), y(t))$ is the position of the moving mass at time $t$. The earth's acceleration of gravity is given by $g$; the pendulum's height is $h$. If we denote the derivative of $x$ and $y$ by $\dot{x}$ and $\dot{y}$ respectively, then, the kinetic energy is given by,

$$
T(\dot{x}, \dot{y})=\frac{1}{2} m(\dot{x}+\dot{y})
$$

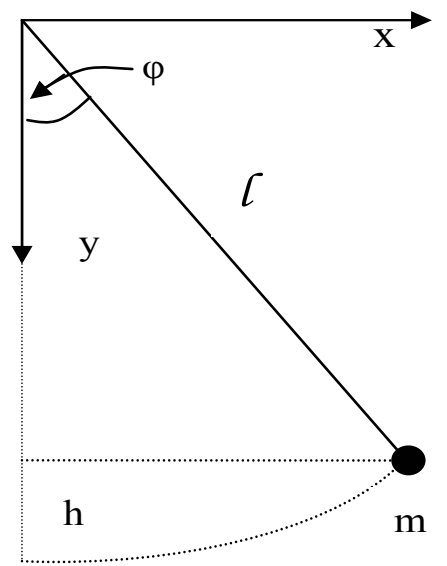

Fig. 1 The mathematical pendulum

The term $(\dot{x}+\dot{y})$ describes the pendulum's velocity. The constraint is found to be

$0=g(x, y)=x^{2}+y^{2}-l^{2}$

Equation (3) - (5) are used to form the Language function

$L(q, \dot{q})=T(\dot{x}, \dot{y})-U(x, y)-\lambda g(x, y)$

Here $q$ denotes the vector $q=(x, y, \lambda)$. Note that $\lambda$ serves as a Language multiplier. The equation of motion is now given by Euler's equations:

$\frac{d}{d t}\left(\frac{d L}{d \dot{q}_{k}}\right)-\frac{d L}{d q_{k}}=0, \quad k=1,2,3 \ldots$.

We arrive at the system

$m \ddot{x}+2 \lambda x=0$

$m \ddot{y}-m g+2 \lambda y=0$

$g(x, y)=0$

By introducing additional variables $u=\dot{x}$ and $v=\dot{y}$ we see that the system of equations (6) - (8) is indeed of the form (1). When solving the above system as an initial value problem, we observe that each initial value $\left(x\left(t_{0}\right), y\left(t_{0}\right)\right)=\left(x_{0}, y_{0}\right)$ has to satisfy the constraint (5) (consistent initialization). No initial condition can be posed for $\lambda$, as $\lambda$ is determined implicitly by the system

$-(8)[1]$.

\section{Some essential concepts for DAEs 3.1 Solvability concepts}

In order to develop a theoretical analysis for the system (1), one has to specify the kind of solution that one is interested, i.e., the solution space in which the solution should lie. Here we discuss the classical (continuously differentiable) solutions[1][4], although other concepts are also available.

\section{Definition:}

Let $X^{k}\left(I, X^{n}\right)$ denote the vector space of all $k$-times continuously differential functions from the real interval $I$ into the complex vector space $C^{n}$.

i. A function $x \in C^{1}\left(I, X^{n}\right)$ is called a solution of (1) if it satisfies (1) point wise.

ii. A function $x \in C^{1}\left(I, X^{n}\right)$ is called a solution of the initial value problem (1) with initial condition $x\left(t_{0}\right)=x_{0} ; t \in I$, if it furthermore satisfies the initial condition.

iii. An initial condition $x\left(t_{0}\right)=x_{0} ; t \in I$, is called consistent with $F$, if the associated initial value problem has at least one solution. In the following, a problem is called solvable if it has at least one solution.[1][5] In most literature, the term solvability is used only for systems which have a unique solution when consistent initial conditions are provided. If the solution of the initial problem is not unique which is, in particular, the case in the context of control problems [1][2], then further conditions have to be specified to single out specific desired solutions.

\subsection{Index concepts}

Since a DAE involves a mixture of differentiation and integrations, one may hope that applying analytical differentiations to a given system and eliminating as needed, repeatedly if necessary, will yield an explicit ODE system for all unknowns. This turns out to be true unless the problem is singular. The number of differentiations needed for this transformation is called the index of the DAE [1]-[5].

In more details, the differentiation index is the minimum number of times that all or part of (1) must be differentiated with respect to $t$ in order to determine $\dot{x}$ as a continuous function of $t$ and $x$. The difference is that an 
ODE has index zero, while an algebraic equation has index one.

\section{Linear DAEs with Constant Co- Efficients}

The linear differential-algebraic equations with constant coefficients are of the form

$E \dot{x}=A x+f(t)$

with $E, A \in X^{m, n}$ and $\quad x \in C\left(I, X^{m}\right)$, possibly together with an initial condition

$x\left(t_{0}\right)=x_{0}$

Such equations occur for example by linearization of autonomous nonlinear problems[4][6] with respect to constant (or critical) solutions where $f$ plays the role of perturbation.

\subsection{Canonical Forms}

The linear differential-algebraic equations with constant coefficients can be treated by purely algebraic techniques. Scaling (9) by a non singular matrix of the form $P \in X^{m, n}$ and the function $x$ according to $x=Q \widetilde{x}$ with nonsingular matrix $Q \in X^{m, n}$, we obtain $\tilde{E} \dot{\tilde{x}}=\tilde{A} \tilde{x}+\tilde{f}(t), \tilde{E}=P E Q, \tilde{A}=P A Q, \tilde{f}=P f$

which is again a linear differential-algebraic equations with constant coefficients.

Moreover, the relation $x=Q \widetilde{x}$ gives a oneto-one correspondence between the corresponding solution sets[1][2]. This means that we can consider the transformed problem (11) instead of (9) with respect to solvability and related questions. The following definition of equivalence is now evident.

\section{Definition}

Two pairs of matrices $\left(E_{i}, A_{i}\right), E_{i}, A_{i} \in P \in X^{m, n}$ are $\quad$ called (strongly) equivalent if there exist nonsingular matrices $P \in X^{m, n}$ and $Q \in X^{n, n}$ such that

$E_{2}=P E_{1} Q, \quad A_{2}=P A_{1} Q$

If this is the case, we write $\left(E_{1}, A_{1}\right):\left(E_{2}, A_{2}\right)$.

Lemma 4.a. The relation induced in Definition canonical form is an equivalence relation.

Theorem 4.A. Let $E, A \in X^{m, n}$, then there exist nonsingular matrices $P \in X^{m, n}$ and $Q \in X^{n, n}$ such that (for all $\lambda \in X$ )
$P(\lambda E-A) Q=\operatorname{diag}\left(\mathfrak{L}_{\epsilon_{1}}, \ldots \ldots, \mathfrak{L}_{\epsilon_{p}}, \mathcal{M}_{\eta_{1}}\right.$ $, \ldots \ldots \ldots, \mathcal{M}_{\eta_{q},}, \mathcal{G}_{\rho_{1}}, \ldots, \ldots, \mathcal{S}_{\rho_{r}}, \quad \mathcal{N}_{\sigma_{l}}$ $\left., \ldots, \mathcal{N}_{\sigma_{s}}\right)$,

where the block entries have the following properties[1]:

i. Every entry $\mathfrak{L}_{\epsilon_{j}}$ is a bidiagonal block of size $\epsilon_{j} \times\left(\epsilon_{j}+1\right), \epsilon_{j} \in \mathbb{N}_{0}$, of the form

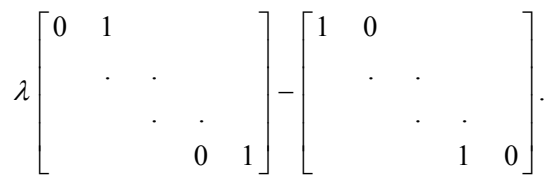

ii. Every entry $\mathcal{M}_{\eta_{1}}$ is a bidiagonal block of size $\left(\eta_{j}+1\right) \times \eta_{j}, \eta_{j} \in \mathbb{N}_{\ominus}$, of the form

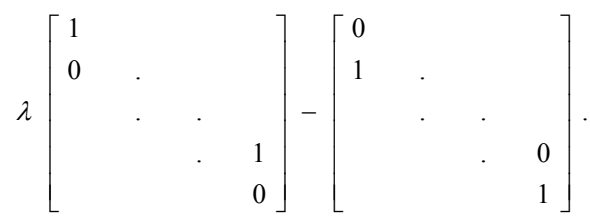

iii. Every entry $\mathcal{S}_{\rho_{j}}$ is a Jordan block of size $\rho_{\mathrm{j}} \times \rho_{\mathrm{j}}, \rho_{\mathrm{j}} \in \mathbb{N}, \lambda_{\mathrm{j}} \in \mathbb{C}$ of the form

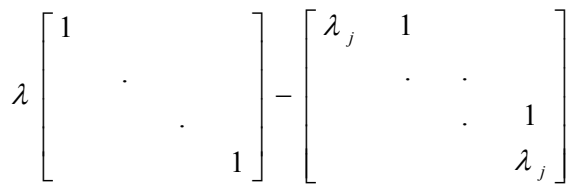

iv. Every entry $N \sigma_{j}$ is a nilpotent block of size $\sigma_{\mathrm{j}} \times \sigma_{\mathrm{j}}, \sigma_{\mathrm{j}} \in \mathbb{N}$, of the form

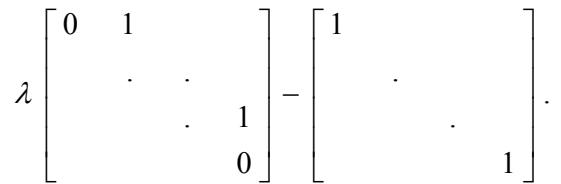

The Kronecker canonical form is unique up to permutation of the blocks,i.e., the kind, size and number of the blocks are characteristic for the matrix pair (E,A).

Note that, the notation for the blocks in Theorem 4.C implies that a pair of $1 \times 1$ matrices $(0,0)$ actually consist of two blocks, a L0 size $0 \times 1$ and a block M0 of size $1 \times 0$.

Example : The Kronecker canonical form of the pair consists of one Jordan block $\mathscr{I}_{1}=\lambda[1]-(1)$, two nilpotent blocks $\mathrm{N}_{2}, \mathrm{~N}_{1}$ and three rectangular blocks L1, L0, M0. 


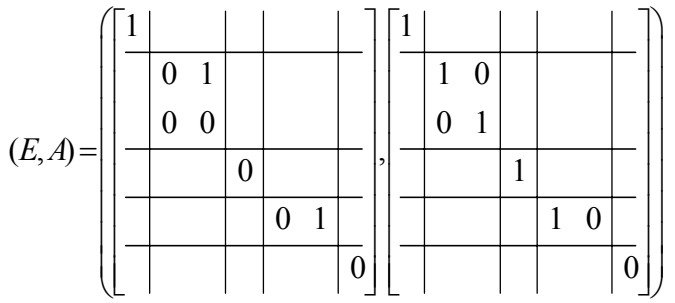

With the help of the Kronecker canonical form, we can now study the behavior of (9) by considering single blocks. An important associated part of the Kronecker canonical form is that of so-called regular matrix pairs[4][5].

\section{Definition}

Let $E, A \in X^{m, n}$. The matrix pair $(E, A)$ is called regular if $m=n$ and the so-called characteristic polynomial $p$ defined by $p(\lambda)=\operatorname{det}(\lambda E A)$

is not the zero polynomial. A matrix pair which is not regular is called singular.

Lemma 4.b.

Every matrix pair which is strongly equivalent to a regular matrix pair is regular.

Proof. We here only to discuss square matrices. Let $E_{2}=P E_{1} Q$ and $A_{2}=P A_{1} Q$ with nonsingular $P$ and $Q$. Then we have,

$$
\begin{aligned}
P_{2}(\lambda) & =\operatorname{det}\left(\lambda E_{2}-A_{2}\right) \\
& =\operatorname{det}\left(\lambda P E_{1} Q-P A_{1} Q\right) \\
& =\operatorname{det} P \operatorname{det}\left(\lambda E_{1}-A_{1}\right) \operatorname{det} Q \\
& =C_{1} \cdot \operatorname{det}\left(\lambda E_{1}-A_{1}\right) \cdot C_{2} \\
& =C P_{1}(\lambda) \quad \text { with } C \neq 0
\end{aligned}
$$

Regularity of a matrix pair is closely related to the solution behavior of the corresponding differential-algebraic equation.

\section{Theorem 4.B.}

Let $E, A \in X^{m, n}$ and $(E, A)$ be regular.

Then we have

$$
(E, A) \sim\left(\left[\begin{array}{cc}
I & 0 \\
0 & N
\end{array}\right],\left[\begin{array}{ll}
J & 0 \\
0 & I
\end{array}\right]\right)
$$

where $J$ is a matrix in Jordan canonical form and $N$ is a nilpotent matrix[1][7][8] also in Jordan canonical form. Moreover, it is allowed that one or the other block is not present.

Proof: Since $(E, A)$ is regular, there exist a $\lambda_{0} \in X$ with $\operatorname{det}\left(\lambda_{0} E-A\right) \neq 0$ implying that $\left(\lambda_{0} E-A\right)$ is non-singular. Hence, $(E, A) \sim\left(E, A-\lambda_{0} E+\lambda_{0} E\right)$

$$
\sim\left(\left(A-\lambda_{0} E\right)^{-1} E, I+\lambda_{0}\left(A-\lambda_{0} E\right)^{-1} E\right)
$$

The next step is to transform $\left(A-\lambda_{0} E\right)^{-1} E$ to Jordan canonical form. This is given by $\operatorname{diag}(\widetilde{J}, \widetilde{N})$, where $\widetilde{J}$ is a nonsingular (i.e., the part corresponding to the nonzero eigenvalues) and $\widetilde{N}$ is a nilpotent, strictly upper triangular matrix. We obtain

$$
(E, A) \sim\left(\left[\begin{array}{cc}
\tilde{J} & 0 \\
0 & \tilde{N}
\end{array}\right],\left[\begin{array}{cc}
I+\lambda_{0} \tilde{J} & 0 \\
0 & I+\lambda_{0} \tilde{N}
\end{array}\right]\right)
$$

Because of the special form of $\widetilde{N}$, the entry $I+\lambda_{0} \tilde{N}$ is a nonsingular upper triangular matrix. It follows that, $(E, A) \sim\left(\left[\begin{array}{cc}I & 0 \\ 0 & \left(I+\lambda_{0} \tilde{N}\right)^{-1} \tilde{N}\end{array}\right],\left[\begin{array}{cc}\tilde{J}^{-1}+\lambda_{0} I & 0 \\ 0 & I\end{array}\right]\right)$ where $\left(I+\lambda_{0} \tilde{N}\right)^{-1} \tilde{N}$ is again a strictly upper triangular matrix and therefore nilpotent. Transformation of the nontrivial entries to Jordan canonical form finally yields (13) with the required block structure[1].

With the help of (13), which is sometimes called Weierstra $\beta$ canonical form, we can now write down the solutions of (9) in the case of a regular matrix pair explicitly.

In particular we utilize that (9) separates into two subproblems when $(E, A)$ is in canonical form. That means, denoting for both subproblems the unknown function by $x \in C^{1}(I, X)$ and the inhomogeneity by $x \in C^{1}\left(I, X^{n}\right)$ and also using Weierstra $\beta$ canonical form, the formula $E \dot{x}=A x+f \quad$ transforms to $\left[\begin{array}{cc}I & 0 \\ 0 & N\end{array}\right]\left[\begin{array}{l}\dot{x}_{1} \\ \dot{x}_{2}\end{array}\right]=\left[\begin{array}{ll}J & 0 \\ 0 & I\end{array}\right]\left[\begin{array}{l}x_{1} \\ x_{2}\end{array}\right]+\left[\begin{array}{l}f_{1} \\ f_{2}\end{array}\right]$

That implies $\quad I \dot{x}_{1}=J x_{1}+f_{1}$,

And $\quad N \dot{x}_{2}=I x_{2}+f_{2}$.

Or, $\quad \dot{x}_{1}=J x_{1}+f_{1}, N \dot{x}_{2}=x_{2}+f_{2}$

(Since $I$ is the identity matrix).

And finally, we get for the first subproblem

$\dot{x}=J x+f(t)$,

which is a linear ordinary differential equation, while for the second subproblem we obtain $N \dot{x}=x+f(t)$.

We here note that in equation (15) $N$ is a nilpotent matrix. When its index of 
nilpotency is zero, it is nothing but a zero matrix. In that case equation (15) is just an algebraic equation of the form as mentioned in equation (2).

We observe that equation (14) is an initial value problem for linear ordinary differential equations and is always solvable. Therefore, here we will only give a more details of (15) $[1][6][8]$.

\section{Lemma 4.c:}

Consider (15) with by $f \in C^{n}\left(I, X^{n}\right), n \geq 1$. Let $v$ be the index of the nil-potency of $N$, i.e., $N^{v} \neq 0$, and $N^{v-1} \neq 0$ Then (15) has the unique solution $x=-\sum_{i=0}^{v-1} N^{i} f^{(i)}$.

Proof : Let $D$ be a linear operator which maps a (continuously) differentiable function $x$ to its derivative $\dot{x}$. Then (15) becomes $N D x=x+f$ $(I-N D) x+f=0$.

or,

Because $N$ is nilpotent and $N$ and $D$ commute $(N$ is constant factor), we obtain $x=-(I-N D)^{-1} f=-\sum_{i=0}^{\infty}(N D)^{i} f=-\sum_{i=0}^{v-1} N^{i} f^{(i)}$

by using the Neumann series.

Inserting into (15), gives

$N \dot{x}-x-f=-N \cdot \sum_{i=0}^{v-1} N^{i} f^{(i+1)}+\sum_{i=0}^{v-1} N^{i} f^{(i)}-f=0$,

or,

$-\sum_{i=0}^{v-1} N^{i+1} f^{(i+1)}+\sum_{i=0}^{v-1} N^{i} f^{i}-f=0$,

thus showing that (16) is indeed a solution.

We can now make two important observations looking at this Lemma. The firestone is, the solution is unique without specifying initial values or, in other words, the only possible initial condition at $t_{0}$ is given by the value $x$ from (16) at $t_{0}$. The second one is, one must require that $f$ is at least $v$ times continuously differentiable to confirm that $x$ is continuously differentiable. Thus we see that, the quantity $v$ plays an important role in the theory of regular DAEs with constant coefficient[6][8].

Definition

Let us consider a pair $(E, A)$ of square matrices that is regular and has a canonical form as in (13). The quantity $v$ defined by
$N^{v}=0, N^{v-1} \neq 0 \quad$ i.e., by the index of nilpotency of $N$ in (13), if the nilpotent block in (13) is present and by $v=0$ if it is absent, is called the index of the matrix pair $(E, A)$, denoted by $v=\operatorname{ind}(E, A)$.

Note that $v$ does not depend on the special transformation to canonical form.

\section{Lemma 4.d.}

Suppose that the pair $(E, A)$ of square matrices has the two canonical forms

$(E, A) \sim\left(\left[\begin{array}{cc}I & 0 \\ 0 & N_{i}\end{array}\right],\left[\begin{array}{cc}J_{i} & 0 \\ 0 & I\end{array}\right]\right), \quad i=1,2$

where $d_{i}, i=1,2$, is the size of the block $J_{i}$. Then $d_{1}=d_{2}$ and, furthermore, $N_{1}^{v}=0$, and $N_{1}^{v-1} \neq 0$ if and only if $N_{2}^{v}=0$ and $N_{2}^{v-1} \neq 0$.

Proof: For the characteristic polynomial [1][5] of two canonical forms, we have

$$
\begin{aligned}
& p_{i}(\lambda)=\operatorname{det}\left(\lambda E_{i}-A_{i}\right) \\
&=\operatorname{det}\left(\lambda\left[\begin{array}{cc}
I & 0 \\
0 & N_{i}
\end{array}\right]-\left[\begin{array}{cc}
J_{i} & 0 \\
0 & I
\end{array}\right]\right) \\
&=\operatorname{det}\left[\begin{array}{cc}
\lambda I-J_{i} & 0 \\
0 & \lambda N_{i}-I
\end{array}\right] \\
&=(-1)^{n-d_{i}} \operatorname{det}\left(\lambda I-J_{i}\right) .
\end{aligned}
$$

Hence, $p_{i}$ is a polynomial of degree $d_{i}$. Since the normal forms are strongly equivalent, $p_{1}$ and $p_{2}$ can only differ by a constant factor according to the proof of Lemma 4.b. Thus $d_{1}=d_{2}$ and the block sizes in the canonical forms are the same. Furthermore, from the strong equivalence [1][4][7] of the canonical forms it follows that there exist nonsingular matric $P=\left[\begin{array}{ll}P_{11} & P_{12} \\ P_{21} & P_{22}\end{array}\right], \quad Q=\left[\begin{array}{ll}Q_{11} & Q_{12} \\ Q_{21} & Q_{22}\end{array}\right]$, partitioned conformably, such that $\left[\begin{array}{ll}P_{11} & P_{12} \\ P_{21} & P_{22}\end{array}\right]\left[\begin{array}{cc}I & 0 \\ 0 & N_{2}\end{array}\right]=\left[\begin{array}{cc}I & 0 \\ 0 & N_{1}\end{array}\right]\left[\begin{array}{ll}Q_{11} & Q_{12} \\ Q_{21} & Q_{22}\end{array}\right]$ and

$\left[\begin{array}{ll}P_{11} & P_{12} \\ P_{21} & P_{22}\end{array}\right]\left[\begin{array}{cc}J_{2} & 0 \\ 0 & I\end{array}\right]=\left[\begin{array}{cc}J_{1} & 0 \\ 0 & I\end{array}\right]\left[\begin{array}{ll}Q_{11} & Q_{12} \\ Q_{21} & Q_{22}\end{array}\right]$. Thus, we obtain the relations 
$P_{11}=Q_{11}, \quad P_{12} N_{2}=Q_{12}, \quad P_{21}=N_{1} Q_{21}, \quad P_{22}=N_{1} Q_{22}$, and

$P_{11} J_{2}=J_{1} Q_{11}, \quad P_{12}=J_{1} Q_{12}, \quad P_{21} J_{2}=Q_{21}, \quad P$

From this, we get $P_{21}=N_{1} P_{21} J_{2}$ and, by successive insertion of $P_{21}$, finally $P_{21}=0$ by the nil potency of $N_{1}$. Similarly, $P_{12}=J_{1} Q_{12}=J_{1} P_{12} N_{2}=0$ due to nilpotency of $N_{2}$ and also $Q_{12}=0=Q_{21}$ for the similar reason. Therefore $P_{11}=Q_{11}$ and $P_{22}=Q_{22}$ must be nonsingular. In particular, $J_{1}$ and $J_{2}$ as well as $N_{1}$ and $N_{2}$ must be similar. That proves our claim, because the Jordan canonical forms of $N_{1}$ and $N_{2}$ consist of the same nilpotent Jordan blocks.

This shows that the block sizes of the canonical form (13) and the index, as defined in Definition, are characteristic values for the pair of square matrices as well as for the associated linear differential-algebraic equations with constant coefficients.

Theorem 4.C.

Let the pair $(E, A)$ of the square matrices be regular and let $P$ and $Q$ be nonsingular matrices which transform (9) and (10) to Weierstra $\beta$ canonical form, [4][8] i.e.,

$P E Q=\left[\begin{array}{cc}I & 0 \\ 0 & N\end{array}\right], \quad P A Q=\left[\begin{array}{cc}J & 0 \\ 0 & I\end{array}\right], \quad P f=\left[\begin{array}{c}\tilde{f}_{1} \\ \tilde{f}_{2}\end{array}\right]$,

and set $Q^{-1} x=\left[\begin{array}{c}\tilde{x}_{1} \\ \tilde{x}_{2}\end{array}\right], \quad Q^{-1} x_{0}=\left[\begin{array}{c}\tilde{x}_{1,0} \\ \tilde{x}_{2,0}\end{array}\right]$.

Furthermore, let $v=\operatorname{ind}(E, A)$ and

$f \in C^{n}\left(I, X^{n}\right)$. Then we have the following:

i. The differential-algebraic equation (9) is solvable.

ii. An initial condition (10) is consistent if and only if $\tilde{x}_{2,0}=-\sum_{i=0}^{\nu-1} N^{i} \tilde{f}_{2}^{(i)}\left(t_{0}\right)$. In particular, the set of consistent initial values $x_{0}$ is nonempty.

iii. Every IVP with consistent initial condition is uniquely solvable

\section{Example:}

Consider the differential-algebraic equation $E \dot{x}=A x+f(t)$ with
$E=\left[\begin{array}{ll|l}0 & 1 & \\ 0 & 0 & \\ \hline & & 0\end{array}\right], \quad A=\left[\begin{array}{ll|l}1 & 0 & \\ 0 & 1 & \\ \hline & & 1\end{array}\right], \quad f(t)=\left[\begin{array}{c}0 \\ -t^{3} \\ \hline-t\end{array}\right]$.

To find the solution set,

$$
x=\left[\begin{array}{l}
x_{1} \\
x_{2} \\
x_{3}
\end{array}\right] \quad \text { and } \quad \dot{x}=\left[\begin{array}{c}
\dot{x}_{1} \\
\dot{x}_{2} \\
\dot{x}_{3}
\end{array}\right]
$$

Therefore, we have from the above system,

$\left[\begin{array}{ll}0 & 1 \\ 0 & 0\end{array}\right] \cdot\left[\begin{array}{l}\dot{x}_{1} \\ \dot{x}_{2}\end{array}\right]=\left[\begin{array}{ll}1 & 0 \\ 0 & 1\end{array}\right] \cdot\left[\begin{array}{l}x_{1} \\ x_{2}\end{array}\right]+\left[\begin{array}{c}0 \\ -t^{3}\end{array}\right]$

and $\quad[0] \cdot\left[\dot{x}_{3}\right]=[1] \cdot\left[x_{3}\right]+[-t]$.

Now, equating the corresponding components and then simplifying we have,

$\left\{\begin{array}{c}\dot{x}_{2}=x_{1}+0 \\ 0=x_{2}+\left(-t^{3}\right)\end{array}\right.$, and $\quad 0=x_{3}-t$.

or, $\left\{\begin{array}{c}\dot{x}_{2}=x_{1} \\ x_{2}=t^{3} \\ x_{3}=t\end{array}\right.$

or, $\left\{\begin{aligned} x_{1}=\frac{d}{d t}\left(x_{2}\right) & =\frac{d}{d t}\left(t^{3}\right)=3 t^{2} \\ x_{2} & =t^{3} \\ x_{3} & =t\end{aligned}\right.$.

Therefore, the solution is unique and given by $x(t)=\left[\begin{array}{lll}3 t^{2} & t^{3} & t\end{array}\right]^{T}$, independent of an initial condition.

\section{Remark}

It remains to consider what happens if a given matrix pair $(E, A)$ is not regular, i.e., if the matrices are not square or the characteristic polynomial (12) vanishes identically. In particular, we want to show that in this case the corresponding initial value problem either has more than one solution or there are arbitrary smooth inhomogeneities for which there is no solution at all [1][5]. With the well-known superposition principle for linear problems we know that two solutions of a inhomogeneous problem[2][8] differ by a solution of the homogeneous problem, this is equivalent to the following statement.

\section{Theorem 4.D.}

Let $E, A \in X^{m, n}$ and suppose that $(E, A)$ is a singular matrix pair. 
i. If $\operatorname{rank}(\lambda E-A)<n$ for all $\lambda \in U$, then the homogeneous initial value problem $E \dot{x}=A x, \quad x\left(t_{0}\right)=0$

has a nontrivial solution.

ii. If rank $(\lambda E-A)=n$ for some $\lambda \in X$ and hence $m>n$, then there exist arbitrarily many smooth inhomogeneities $f$ for which the corresponding differential-algebraic equation is not solvable.

Proof: For the first case, suppose that, $\operatorname{rank}(\lambda E-A)<n \quad$ for $\quad$ some $\lambda \in \quad \amalg$. Let $\lambda_{i}, i=1, \ldots, n+1$, be pair wise different complex numbers. For every $\lambda_{i}$, we have a $v_{i} \in X^{n} \backslash\{0\}$ with $\left(\lambda_{i} E-A\right) v_{i}=0$ and clearly the vector $v_{i}, i=1, \ldots, n+1$, are linearly dependent. Hence, there exist complex number $\alpha_{i}, i=1, \ldots, n+1$, not all zero, such that $\sum_{i=1}^{n+1} \alpha_{i} v_{i}=0$

For the function $x$ defined by

$x(t)=\sum_{i=1}^{n+1} \alpha_{i} v_{i} e^{\lambda_{i}\left(t-t_{0}\right)}$,

we then have $x\left(t_{0}\right)=0$ and

$$
\begin{aligned}
E \dot{x}(t) & =\sum_{i=1}^{n+1} \alpha_{i} \lambda_{i} E v_{i} e^{\lambda_{i}\left(t-t_{0}\right)} \\
& =\sum_{i=1}^{n+1} \alpha_{i} \lambda_{i} E v_{i} e^{\lambda_{i}\left(t-t_{0}\right)}
\end{aligned}
$$

Since $\left(\lambda_{i} E-A\right) v_{i}=0$ and $v_{i} \in X^{n} \backslash\{0\}$,

Therefore, $\left(\lambda_{i} E-A\right)=0$ for $i=1, \ldots ., n+1$.

Hence, equation (4.1.8) gives,

$$
\begin{aligned}
& E \dot{x}(t)=\sum_{i=1}^{n+1} \alpha_{i} A v_{i} e^{\lambda_{i}\left(t-t_{0}\right)} \\
& =A \sum_{i=1}^{n+1} \alpha_{i} v_{i} e^{\lambda_{i}\left(t-t_{0}\right)} \\
& =A x(t) .\left(\text { Since } A \in \coprod^{n \eta} \backslash\{0\}\right. \text { and constant) }
\end{aligned}
$$

Since $x$ is not the zero function, it is a nontrivial solution of the homogeneous initial value problem (4.1.7). [1][4]

For the second case, suppose that there exists an element $\lambda$ such that $\operatorname{rank}(\lambda E-A)=n$. Since $(E, A)$ is assumed to be singular, we have $m>n$.

Now defining the function $x$ by $x(t)=e^{\lambda t} \tilde{x}(t)$,
We have $\dot{x}(t)=e^{\lambda t} \dot{\tilde{x}}(t)+\lambda e^{\lambda t} \tilde{x}(t)$

Then, (4.1) transformed to $E\left(e^{\lambda t} \dot{\tilde{x}}(t)+\lambda e^{\lambda t} \tilde{x}(t)\right)=A e^{\lambda t} \tilde{x}(t)+f(t)$, or, $e^{\lambda t} \cdot E(\dot{\tilde{x}}(t)+\lambda \tilde{x}(t))=A e^{\lambda t} \tilde{x}(t)+f(t)$, or, $E\left(\dot{\tilde{x}}(t)+\lambda \tilde{x}(t)=A \tilde{x}(t)+e^{-\lambda t} f(t)\right.$, or, $E \dot{\tilde{x}}(t)=A \tilde{x}(t)-\lambda \tilde{x}(t)+e^{-\lambda t} f(t)$, or, $E \dot{\tilde{x}}=(A-\lambda E) \tilde{x}+e^{-\lambda t} f(t)$.

Since $A-\lambda E$ has full rank $(=n)$, there exists a nonsingular matrix $P \in \amalg^{m, m}$ such that equation (19), multiplied from the left by $P$, gives

$\left[\begin{array}{l}E_{1} \\ E_{2}\end{array}\right] \dot{\tilde{x}}=\left[\begin{array}{l}I \\ 0\end{array}\right] \tilde{x}+\left[\begin{array}{l}f_{1}(t) \\ f_{2}(t)\end{array}\right]$.

Solving this system of equations, we have

$$
E_{1} \dot{\tilde{x}}=\tilde{x}+f_{1}(t) \text {, and } E_{2} \dot{\tilde{x}}=f_{2}(t) \text {. }
$$

The pair $\left(E_{1}, I\right)$ is regular, implying that $E_{1} \dot{\tilde{x}}=\tilde{x}+f_{1}(t), \quad \tilde{x}\left(t_{0}\right)=\tilde{x}_{0}$

has a unique solution for every sufficiently smooth inhomogeneity $f_{1}$ and for every consistent initial value. But then $f_{2}(t)=E_{2} \dot{\tilde{x}}(t)$ is a consistency condition for the inhomogeneity $f_{2}$ that must hold for a solution to exist. But there exist arbitrarily many smooth functions $f$ for which this consistency condition is not satisfied. [4][6][7]

Example :

Consider the differential-algebraic equation $E \dot{x}=A x+f(t)$ with,

$E=\left[\begin{array}{ll|l}0 & 1 & \\ & & 1 \\ & & 0\end{array}\right], \quad A=\left[\begin{array}{ll|l}1 & 0 & \\ & & 0 \\ & & 1\end{array}\right], \quad f=\left[\begin{array}{l}f_{1} \\ f_{2} \\ f_{3}\end{array}\right]$.

The solution is derived as follows:

Let $\tilde{E}_{1}=\left[\begin{array}{ll}0 & 1\end{array}\right], \quad \tilde{A}_{1}=\left[\begin{array}{ll}1 & 0\end{array}\right], \quad \tilde{f}_{1}=\left[f_{1}\right]$ and $\tilde{E}_{2}=\left[\begin{array}{l}1 \\ 0\end{array}\right], \quad \tilde{A}_{2}=\left[\begin{array}{l}0 \\ 1\end{array}\right], \quad \tilde{f}_{2}=\left[\begin{array}{l}f_{2} \\ f_{3}\end{array}\right]$.

Here the $\operatorname{pair}\left(\tilde{E}_{1}, \tilde{A}_{1}\right)=\left(\left[\begin{array}{ll}0 & 1\end{array}\right],\left[\begin{array}{ll}1 & 0\end{array}\right]\right)$ consists of the rectangular block L1, and the $\operatorname{pair}\left(\tilde{E}_{2}, \tilde{A}_{2}\right)=\left(\left[\begin{array}{l}1 \\ 0\end{array}\right],\left[\begin{array}{l}0 \\ 1\end{array}\right]\right)$ consists of the bidiagonal block $M_{l}$.

To find the solution set, 


$$
x=\left[\begin{array}{c}
x_{1} \\
x_{2} \\
x_{3}
\end{array}\right] \quad \text { and } \quad \dot{x}=\left[\begin{array}{c}
\dot{x}_{1} \\
\dot{x}_{2} \\
\dot{x}_{3}
\end{array}\right]
$$

\section{Case I:}

For the homogeneous problem (17), we have,

$$
\begin{aligned}
& {\left[\begin{array}{ll}
0 & 1
\end{array}\right] \cdot\left[\begin{array}{l}
\dot{x}_{1} \\
\dot{x}_{2}
\end{array}\right]=\left[\begin{array}{ll}
1 & 0
\end{array}\right] \cdot\left[\begin{array}{l}
x_{1} \\
x_{2}
\end{array}\right] \text {, and }} \\
& {\left[\begin{array}{l}
1 \\
0
\end{array}\right] \cdot\left[\dot{x}_{3}\right]=\left[\begin{array}{l}
0 \\
1
\end{array}\right] \cdot\left[x_{3}\right] .}
\end{aligned}
$$

Solving the above systems, we get, $\dot{x}_{2}=x_{1}, \dot{x}_{3}=0$, and $0=x_{3}$.

This shows that the homogeneous problem (17) has non-trivial solution.

\section{Case II:}

For the differential-algebraic equation $E \dot{x}=A x+f(t)$, we have the forms,

$$
\begin{aligned}
& {\left[\begin{array}{ll}
0 & 1
\end{array}\right] \cdot\left[\begin{array}{l}
\dot{x}_{1} \\
\dot{x}_{2}
\end{array}\right]=\left[\begin{array}{ll}
1 & 0
\end{array}\right] \cdot\left[\begin{array}{l}
x_{1} \\
x_{2}
\end{array}\right]+\left[f_{1}\right] \quad \text { and }} \\
& {\left[\begin{array}{l}
1 \\
0
\end{array}\right] \cdot\left[\dot{x}_{3}\right]=\left[\begin{array}{l}
0 \\
1
\end{array}\right] \cdot\left[x_{3}\right]+\left[\begin{array}{l}
f_{2} \\
f_{3}
\end{array}\right] \cdot} \\
& \text { Solving this systems, we get } \\
& \dot{x}_{2}=x_{1}+f_{1}, \dot{x}_{3}=f_{2} \text { and } x_{3}=-f_{3}
\end{aligned}
$$

We here observe that for

Case III: the solution is independent of initial values. For the solution to exist, we need $f_{2}=-\dot{f}_{3}$. The solution is not unique, since any continuous differentiable function $x_{1}$ can be chosen.

\section{Conclusion}

Starting with a new concept of DAE systems, we have discussed the system with canonical form and some examples regarding the uniqueness and existence of the solutions. This canonical form is a powerful tool in the analysis of circuit equations and dynamical systems arising in practical case. There has been a tremendous explosion in the research literature in the area of differential-algebraic equations.

\section{Acknowledgement}

The authors are grateful to Prof. Dr. Peter Benner, Head of research unit Mathematik in Industrie und Technik TU Chemnitz, and Prof. Md. Abdul Malek, Department of
Mathematics, Jahangirnagar University, for their valuable suggestions.

\section{References}

[1] Peter Kunkel, Volker Mehrmann, "DifferentialAlgebraic Equations analysis and numerical solution", European Mathematical Society, Swizerland, 2006.

[2] Kunkel,P, Mehrmann,V, "Analysis and Numerical linear differential algebraic equations" Chemnitz University of Technology.Department of Mathematics, preprint SPG 94-27(1994).

[3] Denk, G., Winkler, R., "Modelling and simulation of transient noise in circuit simulation", International Journal of Mathematical and Computational Modelling of Dynamical Systems(MCMDS), Vol. 13, No. 4, 2007, pp. 383394.

[4] Gear, C.W.; Petzold, L., "ODE methods for solution of differential algebraic systems", SIAM Journal of Numerical Analysis, vol. 21, No. 4, pp. 716-728, 1984

[5] Hairer, E. Wanner, G., "Solving ordinary differential equations II: stiff and differential algebraic problems", Springer Series in Computational Mathematics, Vol. 14, 2004.

[6] Petzold, L, "Differential / algebraic equations are not ODE", SIAM Journal on Scientific Computing, Vol 3, No. 1, pp. 367-384, 1982.

[7] Sleffen Schulz, "Four lectures on Differential algebraic equations", Humboldt Universität, Berlin., Research Report Series 497, 2003.

[8] Butcher,J.C., "Numerical Methods for Ordinary Differential Equations", John Wiley and Sons, 2003.

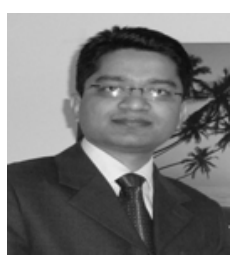

M. Sahadet Hossain is a Ph.D candidate of the Dept. of Mathematics, TU Chemnitz, Germany. He obtained his Bachelor and Masters in Mathematics from University of Dhaka, Bangladesh. His research interest is control theory. Now he is working on model reduction of linear timevarying descriptor systems.

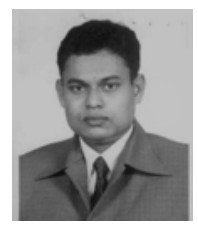

M. Mostafizur Rahman is doing masters in11 computer science in University of Trento, Italy. He has obtained his Bachelor and Masters in Mathematics from University of Dhaka, Bangladesh. His research interest is BioMathematics. Now he is working on mathematical model of biological components. 